\title{
UNIVERSAL BOUNDS FOR EIGENVALUES OF A BUCKLING PROBLEM II
}

\author{
QING-MING CHENG* AND HONGCANG YANG**
}

\begin{abstract}
In this paper, we investigate universal estimates for eigenvalues of a buckling problem. For a bounded domain in a Euclidean space, we solve partially a conjecture proposed in [7. For a domain in the unit sphere, we give an important improvement on the results of Wang and Xia [16].
\end{abstract}

\section{INTRODUCTION}

Let $M$ be an $n$-dimensional complete Riemannian manifold and $\Omega \subset M$ a bounded domain in $M$ with piecewise smooth boundary $\partial \Omega$. A Dirichlet eigenvalue problem of Laplacian is given by

$$
\begin{cases}\triangle u=-\lambda u, & \text { in } \Omega, \\ u=0, & \text { on } \partial \Omega,\end{cases}
$$

which is also called a fixed membrane problem, where $\Delta$ denotes the Laplacian on $M$. The spectrum of this eigenvalue problem is real and discrete:

The following eigenvalue problem of a biharmonic operator is called a buckling problem:

$$
\left\{\begin{array}{l}
\Delta^{2} u=-\Lambda \Delta u \text { in } \Omega \\
\left.u\right|_{\partial \Omega}=\left.\frac{\partial u}{\partial \nu}\right|_{\partial \Omega}=0
\end{array}\right.
$$

which describes the critical buckling load of a clamped plate subjected to a uniform compressive force around its boundary, where $\nu$ is the outward unit normal vector field of the boundary $\partial \Omega$. It is known that the spectrum of the buckling problem is also real and discrete.

Key words and phrases: universal estimates for eigenvalues, a biharmonic operator and a buckling problem.

2001 Mathematics Subject Classification: 35P15, 58G25, 53C42.

* Research partially Supported by a Grant-in-Aid for Scientific Research from the Japan Society for the Promotion of Science.

** Research partially Supported by SF of CAS. 
When $\Omega \subset \mathbf{R}^{n}$ be a bounded domain in an $n$-dimensional Euclidean space $\mathbf{R}^{n}$, Payne, Pólya and Weinberger [14] and [15] proved the following inequality for eigenvalues of the eigenvalue problem (1.1): for $k=1,2, \cdots$,

$$
\lambda_{k+1}-\lambda_{k} \leq \frac{4}{k n} \sum_{i=1}^{k} \lambda_{i}
$$

One calls it a universal inequality since it does not depend on the domain $\Omega$.

On the other hand, Payne, Pólya and Weinberger [14] and [15] also studied eigenvalues of the buckling problem for a bounded domain $\Omega$ in $\mathbf{R}^{n}$ and intended to derive a universal inequality for eigenvalues of the buckling problem. But it is very hard to deal with this problem. They only proved, for $n=2$,

$$
\Lambda_{2} \leq 3 \Lambda_{1}
$$

As an open problem, Payne, Pólya and Weinberger [14 and [15] proposed the following:

Problem. Whether can one obtain a universal inequality for eigenvalues of the buckling problem (1.2) on a bounded domain in a Euclidean space, which is similar to the universal inequality (1.3) for the eigenvalues of the fixed membrane problem (1.1)?

Although many mathematicians have intended to attack this problem, there are no any progresses except for lower order eigenvalues. For lower order eigenvalues, Hile and Yeh [13] and so on improved the result of Payne, Pólya and Weinberger to

$$
\Lambda_{2} \leq \frac{n^{2}+8 n+20}{(n+2)^{2}} \Lambda_{1}
$$

Furthermore, Ashbaugh [2] (cf. [1]) has obtained

$$
\sum_{i=1}^{n} \Lambda_{i+1} \leq(n+4) \Lambda_{1}
$$

and he has commented that to obtain a universal inequality for eigenvalues of the buckling problem remains a challenge for mathematicians since 1955.

Recently, by introducing a new method to construct trial functions for the buckling problem, Cheng and Yang [7] have obtained the following universal inequality for eigenvalues of the buckling problem (1.2):

$$
\sum_{i=1}^{k}\left(\Lambda_{k+1}-\Lambda_{i}\right)^{2} \leq \frac{4(n+2)}{n^{2}} \sum_{i=1}^{k}\left(\Lambda_{k+1}-\Lambda_{i}\right) \Lambda_{i} .
$$

Thus, the problem proposed by Payne, Pólya and Weinberger has been solved affirmatively. Furthermore, it is very important to prove a sharp universal inequality for eigenvalues of the buckling problem. The following has been conjectured in [7]: 
Conjecture. Eigenvalues of the buckling problem on a bounded domain in a Euclidean space $\mathbf{R}^{n}$ satisfy the following universal inequality:

$$
\sum_{i=1}^{k}\left(\Lambda_{k+1}-\Lambda_{i}\right)^{2} \leq \frac{4}{n} \sum_{i=1}^{k}\left(\Lambda_{k+1}-\Lambda_{i}\right) \Lambda_{i}
$$

The first purpose in this paper is to attack the above conjecture, we prove the following:

Theorem 1.1. Let $\Lambda_{i}$ be the $i$-th eigenvalue of the buckling problem (1.2) for a bounded domain $\Omega \subset \mathbf{R}^{n}$. Then, we have

$$
\sum_{i=1}^{k}\left(\Lambda_{k+1}-\Lambda_{i}\right)^{2} \leq \frac{4\left(n+\frac{4}{3}\right)}{n^{2}} \sum_{i=1}^{k}\left(\Lambda_{k+1}-\Lambda_{i}\right) \Lambda_{i}
$$

Remark 1.1. Since our universal inequality is a quadratic inequality of the eigenvalue $\Lambda_{k+1}$, we can conclude an upper bound of $\Lambda_{k+1}$ and an upper bound of the gap between two consecutive eigenvalues as in [7] from (1.5). We will not give it in details.

When $M$ is an $n$-dimensional unit sphere $S^{n}(1)$, Wang and Xia [16] have studied the buckling problem for a domain $\Omega$ in $S^{n}(1)$. They have obtained a universal inequality for eigenvalues of the buckling problem, namely, they have proved that eigenvalues of the buckling problem (1.2) for a domain $\Omega$ in the unit sphere $S^{n}(1)$ satisfy

$$
\begin{aligned}
& 2 \sum_{i=1}^{k}\left(\Lambda_{k+1}-\Lambda_{i}\right)^{2} \\
& \leq \sum_{i=1}^{k}\left(\Lambda_{k+1}-\Lambda_{i}\right)^{2}\left\{\delta \Lambda_{i}+\frac{\delta^{2}\left(\Lambda_{i}-(n-2)\right)}{4\left(\delta \Lambda_{i}+n-2\right)}\right\} \\
& +\frac{1}{\delta} \sum_{i=1}^{k}\left(\Lambda_{k+1}-\Lambda_{i}\right)\left(\Lambda_{i}+\frac{(n-2)^{2}}{4}\right),
\end{aligned}
$$

where $\delta$ is an arbitrary positive constant.

The second purpose in this paper is to give an important improvement for the result of Wang and Xia. 
Theorem 1.2. Eigenvalues $\Lambda_{i}$ 's of the buckling problem (1.2) for a domain $\Omega$ in the unit sphere $S^{n}(1)$ satisfy

$$
\begin{aligned}
& 2 \sum_{i=1}^{k}\left(\Lambda_{k+1}-\Lambda_{i}\right)^{2}+(n-2) \sum_{i=1}^{k} \frac{\left(\Lambda_{k+1}-\Lambda_{i}\right)^{2}}{\Lambda_{i}-(n-2)} \\
& \leq \sum_{i=1}^{k}\left(\Lambda_{k+1}-\Lambda_{i}\right)^{2}\left\{\Lambda_{i}-\frac{n-2}{\Lambda_{i}-(n-2)}\right\} \delta_{i} \\
& +\sum_{i=1}^{k} \frac{\left(\Lambda_{k+1}-\Lambda_{i}\right)}{\delta_{i}}\left(\Lambda_{i}+\frac{(n-2)^{2}}{4}\right)
\end{aligned}
$$

for an arbitrary positive non-increasing monotone sequence $\left\{\delta_{i}\right\}_{i=1}^{k}$.

Remark 1.2. It is obvious that our result is sharper than one of Wang and Xia [16] even if we take $\delta_{i}=\delta$ for any $i$. Since our universal inequality is a quadratic inequality of $\Lambda_{k+1}$, we can obtain an explicit upper bound for the eigenvalue $\Lambda_{k+1}$ from (1.7).

In particular, when $n=2$, we have

Corollary 1.1. Eigenvalues $\Lambda_{i}$ 's of the buckling problem (1.2) for a domain $\Omega$ in the unit sphere $S^{2}(1)$ satisfy

$$
\sum_{i=1}^{k}\left(\Lambda_{k+1}-\Lambda_{i}\right)^{2} \leq \sum_{i=1}^{k}\left(\Lambda_{k+1}-\Lambda_{i}\right) \Lambda_{i}^{2} .
$$

Proof. Since $n=2$, from the theorem 1.2 and taking $\delta_{i}=\frac{1}{\Lambda_{i}}$, for $i=1,2, \cdots, k$, for which $\left\{\delta_{i}\right\}_{i=1}^{k}$ is a positive non-increasing monotone sequence, we finish the proof of the corollary 1.1 .

Remark 1.3. About the recent developments in universal inequalities for eigenvalues of the Dirichlet eigenvalue problem of the Laplacian and the clamped plate problem, readers can see [3], [4], [5], 6], [8], [11], [12] and [17].

\section{Proof of the theorem 1.1}

For the convenience of readers, we review the method for constructing trial functions introduced by Cheng and Yang [7]. In this section, $\Omega$ is assumed to be a bounded domain in $\mathbf{R}^{n}$. For functions $f$ and $h$, we define Dirichlet inner product $(f, h)_{D}$ of $f$ and $h$ by

$$
(f, h)_{D}=\int_{\Omega}\langle\nabla f, \nabla h\rangle .
$$

Dirichlet norm of a function $f$ is defined by

$$
\|f\|_{D}=\left\{(f, f)_{D}\right\}^{1 / 2}=\left(\int_{\Omega} \sum_{\alpha=1}^{n}\left|\nabla_{\alpha} f\right|^{2}\right)^{1 / 2} .
$$


Let $u_{i}$ be the $i$-th orthonormal eigenfunction of the buckling problem (1.2) corresponding to the eigenvalue $\Lambda_{i}$, namely, $u_{i}$ satisfies

$$
\left\{\begin{array}{l}
\Delta^{2} u_{i}=-\Lambda_{i} \Delta u_{i} \quad \text { in } \Omega \\
\left.u_{i}\right|_{\partial \Omega}=\left.\frac{\partial u_{i}}{\partial \nu}\right|_{\partial \Omega}=0 \\
\left(u_{i}, u_{j}\right)_{D}=\int_{\Omega}\left\langle\nabla u_{i}, \nabla u_{j}\right\rangle=\delta_{i j}
\end{array}\right.
$$

$H_{2}^{2}(\Omega)$ defined by

$$
H_{2}^{2}(\Omega)=\left\{f: f, \nabla_{\alpha} f, \nabla_{\alpha} \nabla_{\beta} f \in L^{2}(\Omega), \quad \alpha, \beta=1, \ldots, n\right\}
$$

is a Hilbert space with norm $\|\cdot\|_{2}$ :

$$
\|f\|_{2}=\left(\int_{\Omega}|f|^{2}+\int_{\Omega}|\nabla f|^{2}+\sum_{\beta, \alpha=1}^{n}\left(\nabla_{\alpha} \nabla_{\beta} f\right)^{2}\right)^{1 / 2} .
$$

Let $H_{2, D}^{2}(\Omega)$ be a subspace of $H_{2}^{2}(\Omega)$ defined as

$$
H_{2, D}^{2}(\Omega)=\left\{f \in H_{2}^{2}(\Omega):\left.f\right|_{\partial M}=\left.\frac{\partial}{\partial \nu} f\right|_{\partial \Omega}=0\right\} .
$$

The biharmonic operator $\Delta^{2}$ defines a self-adjoint operator acting on $H_{2, D}^{2}(\Omega)$ with discrete eigenvalues $\left\{0<\Lambda_{1} \leq \Lambda_{2} \leq \cdots \leq \Lambda_{k} \leq \cdots\right\}$ for the buckling problem (1.2) and the eigenfunctions defined in (2.1)

$$
\left\{u_{i}\right\}_{i=1}^{\infty}=\left\{u_{1}, u_{2}, \cdots, u_{k}, \cdots\right\}
$$

form a complete orthogonal basis for Hilbert space $H_{2, D}^{2}(\Omega)$. We define an inner product $(\mathbf{f}, \mathbf{h})$ for vector-valued functions $\mathbf{f}=\left(f^{1}, f^{2}, \cdots, f^{n}\right) \in \mathbf{R}^{n}$ and $\mathbf{h}=$ $\left(h^{1}, h^{2}, \cdots, h^{n}\right) \in \mathbf{R}^{n}$ by

$$
(\mathbf{f}, \mathbf{h}) \equiv \int_{\Omega}\langle\mathbf{f}, \mathbf{h}\rangle=\int_{\Omega} \sum_{\alpha=1}^{n} f^{\alpha} h^{\alpha} .
$$

The norm of $\mathbf{f}$ is defined by

$$
\|\mathbf{f}\|=(\mathbf{f}, \mathbf{f})^{1 / 2}=\left\{\int_{\Omega} \sum_{\alpha=1}^{n}\left(f^{\alpha}\right)^{2}\right\}^{1 / 2} .
$$

Denote a Hilbert space $\mathbf{H}_{1}^{2}(\Omega)$ of the vector-valued functions as

$$
\mathbf{H}_{1}^{2}(\Omega)=\left\{\mathbf{f}: f^{\alpha}, \nabla_{\beta} f^{\alpha} \in L^{2}(\Omega), \text { for } \alpha, \beta=1, \ldots, n\right\}
$$

with norm $\|\cdot\|_{1}$ :

$$
\|\mathbf{f}\|_{1}=\left(\|\mathbf{f}\|^{2}+\int_{\Omega} \sum_{\alpha, \beta=1}^{n}\left|\nabla_{\alpha} f^{\beta}\right|^{2}\right)^{1 / 2}
$$

Let $\mathbf{H}_{1, D}^{2}(\Omega) \subset \mathbf{H}_{1}^{2}(\Omega)$ be a subspace of $\mathbf{H}_{1}^{2}(\Omega)$ spanned by the vector-valued functions $\left\{\nabla u_{i}\right\}_{i=1}^{\infty}$, which form a complete orthonormal basis of $\mathbf{H}_{1, D}^{2}(\Omega)$.

It is easy to see that for any $f \in H_{2, D}^{2}(\Omega), \nabla f \in \mathbf{H}_{1, D}^{2}(\Omega)$ and for any $\mathbf{h} \in \mathbf{H}_{1, D}^{2}(\Omega)$, there exists a function $f \in H_{2, D}^{2}(\Omega)$ such that $\mathbf{h}=\nabla f$. 
Let $x^{p}$ for $p=1,2, \cdots, n$ be the $p$-th coordinate function of $\mathbf{R}^{n}$. For the vectorvalued function $x^{p} \nabla u_{i}, i=1, \ldots, k$, we decompose it into

$$
x^{p} \nabla u_{i}=\nabla h_{p i}+\mathbf{w}_{p i},
$$

where $h_{p i} \in H_{2, D}^{2}(\Omega)$ and $\nabla h_{p i}$ is the projection of $x^{p} \nabla u_{i}$ onto $\mathbf{H}_{1, D}^{2}(\Omega)$ and $\mathbf{w}_{p i} \perp$ $H_{1, D}^{2}(\Omega)$. Thus,

$$
\left(\mathbf{w}_{p i}, \nabla u\right)=\int_{\Omega} \sum_{j=1}^{n} w_{p i}^{j} \nabla_{j} u=0, \text { for any } u \in H_{2, D}^{2}(\Omega) .
$$

Therefore, since $H_{2, D}^{2}(\Omega)$ is dense in $L^{2}(\Omega)$ and $C^{1}(\Omega)$ is dense in $L^{2}(\Omega)$, we have, for any function $h \in C^{1}(\Omega) \cap L^{2}(\Omega)$,

$$
\left(\mathbf{w}_{p i}, \nabla h\right)=0 \text {. }
$$

Hence, from the definition of $\mathbf{w}_{p i}$ and (2.4), we have

$$
\left\{\begin{array}{l}
\left.\mathbf{w}_{p i}\right|_{\partial \Omega}=0, \\
\left\|\operatorname{div} \mathbf{w}_{p i}\right\|^{2}=0, \quad\left(\operatorname{div} \mathbf{w}_{p i} \equiv \sum_{j=1}^{n} \nabla_{j} w_{p i}^{j}\right) .
\end{array}\right.
$$

We define function $\varphi_{p i}$ by

$$
\varphi_{p i}=h_{p i}-\sum_{j=1}^{k} b_{p i j} u_{j}
$$

where

$$
b_{p i j}=\int x^{p}\left\langle\nabla u_{i}, \nabla u_{j}\right\rangle=b_{p j i} .
$$

It is easy to check, from the definition (2.2) of $h_{p i}$, that $\varphi_{p i}$ satisfies

$$
\left.\varphi_{p i}\right|_{\partial \Omega}=\left.\frac{\partial \varphi_{p i}}{\partial \nu}\right|_{\partial \Omega}=0 \text { and }\left(\varphi_{p i}, u_{j}\right)_{D}=\left(\nabla \varphi_{p i}, \nabla u_{j}\right)=0,
$$

for any $j=1,2, \cdots, k$. Hence, we know that $\varphi_{p i}$ is a trial function.

In order to prove our theorem 1.1, we prepare three lemmas.

Lemma 2.1. For any $p$ and $i$, we have

$$
1+2\left\|\left\langle\nabla x^{p}, \nabla u_{i}\right\rangle\right\|^{2}=2 \int x^{p} u_{i}\left\langle\nabla x^{p}, \nabla\left(\Delta u_{i}\right)\right\rangle .
$$

Proof. From the Stokes' formula, we have

$$
\begin{aligned}
& \int\left\langle x^{p} u_{i} \nabla x^{p}, \nabla\left(\Delta u_{i}\right)\right\rangle \\
& =-\int \operatorname{div}\left(x^{p} u_{i} \nabla x^{p}\right) \Delta u_{i} \\
& =-\int u_{i} \Delta u_{i}-\int x^{p} \Delta u_{i}\left\langle\nabla x^{p}, \nabla u_{i}\right\rangle,
\end{aligned}
$$




$$
\begin{aligned}
& \int x^{p} \Delta u_{i}\left\langle\nabla x^{p}, \nabla u_{i}\right\rangle \\
& =-\int\left\langle\nabla x^{p}, \nabla u_{i}\right\rangle^{2}-\int x^{p}\left\langle\nabla u_{i}, \nabla\left\langle\nabla x^{p}, \nabla u_{i}\right\rangle\right\rangle \\
& =-\left\|\left\langle\nabla x^{p}, \nabla u_{i}\right\rangle\right\|^{2}+\int \operatorname{div}\left(x^{p} \nabla\left\langle\nabla x^{p}, \nabla u_{i}\right\rangle\right) u_{i} \\
& =-\left\|\left\langle\nabla x^{p}, \nabla u_{i}\right\rangle\right\|^{2}+\int\left\langle\nabla x^{p}, \nabla\left\langle\nabla x^{p}, \nabla u_{i}\right\rangle\right\rangle u_{i}+\int x^{p} u_{i} \Delta\left\langle\nabla x^{p}, \nabla u_{i}\right\rangle \\
& =-\left\|\left\langle\nabla x^{p}, \nabla u_{i}\right\rangle\right\|^{2}-\int\left\langle\nabla x^{p}, \nabla u_{i}\right\rangle^{2}+\int x^{p} u_{i}\left\langle\nabla x^{p}, \nabla\left(\Delta u_{i}\right)\right\rangle .
\end{aligned}
$$

Since $\left\|\nabla u_{i}\right\|^{2}=1$, we have

$$
1+2\left\|\left\langle\nabla x^{p}, \nabla u_{i}\right\rangle\right\|^{2}=2 \int x^{p} u_{i}\left\langle\nabla x^{p}, \nabla\left(\Delta u_{i}\right)\right\rangle .
$$

According to $x^{p} \nabla u_{i}=\nabla h_{p i}+\mathbf{w}_{p i}$ and $\nabla\left(x^{p} u_{i}\right) \in H_{1, D}^{2}(\Omega)$, we have

$$
u_{i} \nabla x^{p}=\nabla\left(x^{p} u_{i}\right)-\nabla h_{p i}-\mathbf{w}_{p i}=\nabla q_{p i}-\mathbf{w}_{p i}
$$

with $\nabla q_{p i}=\nabla\left(x^{p} u_{i}\right)-\nabla h_{p i}$ and $q_{p i} \in H_{2, D}^{2}(\Omega)$. Hence, we derive

$$
\left\|u_{i}\right\|^{2}=\left\|\nabla q_{p i}\right\|^{2}+\left\|\mathbf{w}_{p i}\right\|^{2} .
$$

Lemma 2.2. For any $p$ and $i$,

$$
3\left\|\left\langle\nabla x^{p}, \nabla u_{i}\right\rangle\right\|^{2}-2 \Lambda_{i}\left\|\nabla q_{p i}\right\|^{2}=\frac{1}{2}-\frac{1}{2} \Lambda_{i}\left\|u_{i}\right\|^{2} .
$$

Proof. Since, from the Stokes' formula,

$$
\begin{aligned}
& \int x^{p} u_{i}\left\langle\nabla x^{p}, \nabla\left(\Delta u_{i}\right)\right\rangle \\
= & \int \Delta\left(x^{p} u_{i}\right)\left\langle\nabla x^{p}, \nabla u_{i}\right\rangle \\
= & -\int\left\langle u_{i} \nabla x^{p}, \nabla\left(\Delta\left(x^{p} u_{i}\right)\right)\right\rangle \\
= & -\int\left\langle\nabla q_{p i}, \nabla\left(\Delta\left(x^{p} u_{i}\right)\right)\right\rangle \quad(\text { from }(2.4) \text { and }(2.9)) \\
= & \int q_{p i} \Delta^{2}\left(x^{p} u_{i}\right) \\
= & \int q_{p i}\left(4\left\langle\nabla x^{p}, \nabla\left(\Delta u_{i}\right)\right\rangle-\Lambda_{i} x^{p} \Delta u_{i}\right) \\
= & -4 \int \Delta u_{i}\left\langle\nabla q_{p i}, \nabla x^{p}\right\rangle-\Lambda_{i} \int q_{p i} x^{p} \Delta u_{i}
\end{aligned}
$$


and

$$
\begin{aligned}
& -\Lambda_{i} \int q_{p i} x^{p} \Delta u_{i} \\
& =\Lambda_{i} \int\left\langle\nabla q_{p i}, x^{p} \nabla u_{i}\right\rangle+\Lambda_{i} \int q_{p i}\left\langle\nabla x^{p}, \nabla u_{i}\right\rangle \\
& =\Lambda_{i} \int\left\langle\nabla q_{p i}, x^{p} \nabla u_{i}\right\rangle-\Lambda_{i} \int\left\langle\nabla q_{p i}, u_{i} \nabla x^{p}\right\rangle \\
& =\Lambda_{i} \int\left\langle\nabla q_{p i}, x^{p} \nabla u_{i}\right\rangle-\Lambda_{i}\left\|\nabla q_{p i}\right\|^{2}, \\
& \quad-4 \int \Delta u_{i}\left\langle\nabla q_{p i}, \nabla x^{p}\right\rangle \\
& =-4 \int\left\langle\nabla\left(\Delta q_{p i}\right), u_{i} \nabla x^{p}\right\rangle \\
& =4 \int \Delta q_{p i}\left\langle\nabla x^{p}, \nabla u_{i}\right\rangle \\
& =-4 \int\left\langle\nabla q_{p i}, \nabla\left\langle\nabla x^{p}, \nabla u_{i}\right\rangle\right\rangle \\
& =-4 \int\left\langle u_{i} \nabla x^{p}, \nabla\left\langle\nabla x^{p}, \nabla u_{i}\right\rangle\right\rangle \\
& =4\left\|\left\langle\nabla x^{p}, \nabla u_{i}\right\rangle\right\|^{2},
\end{aligned}
$$

we obtain

$$
\int x^{p} u_{i}\left\langle\nabla x^{p}, \nabla\left(\Delta u_{i}\right)\right\rangle=4\left\|\left\langle\nabla x^{p}, \nabla u_{i}\right\rangle\right\|^{2}+\Lambda_{i} \int\left\langle\nabla q_{p i}, x^{p} \nabla u_{i}\right\rangle-\Lambda_{i}\left\|\nabla q_{p i}\right\|^{2} .
$$

From the lemma 2.1 and the above equality, we have

$$
6\left\|\left\langle\nabla x^{p}, \nabla u_{i}\right\rangle\right\|^{2}-2 \Lambda_{i}\left\|\nabla q_{p i}\right\|^{2}-1=-2 \Lambda_{i} \int\left\langle\nabla q_{p i}, x^{p} \nabla u_{i}\right\rangle .
$$

Furthermore, from (2.4), $x^{p} \nabla u_{i}=\nabla h_{p i}+\mathbf{w}_{p i}$ and $\nabla q_{p i}=\nabla\left(x^{p} u_{i}\right)-\nabla h_{p i}$, we have

$$
\begin{aligned}
& \int\left\langle\nabla q_{p i}, x^{p} \nabla u_{i}\right\rangle \\
= & \int\left\langle\nabla q_{p i}, \nabla h_{p i}\right\rangle \\
= & \int\left\langle\nabla q_{p i}, \nabla\left(x^{p} u_{i}\right)-\nabla q_{p i}\right\rangle \\
= & \int\left\langle\nabla q_{p i}, \nabla\left(x^{p} u_{i}\right)\right\rangle-\left\|\nabla q_{p i}\right\|^{2} \\
= & \int\left\langle u_{i} \nabla x^{p}, \nabla\left(x^{p} u_{i}\right)\right\rangle-\left\|\nabla q_{p i}\right\|^{2} \\
= & \left\|u_{i}\right\|^{2}+\int\left\langle u_{i} \nabla x^{p}, x^{p} \nabla u_{i}\right\rangle-\left\|\nabla q_{p i}\right\|^{2} .
\end{aligned}
$$


Since

$$
\int\left\langle u_{i} \nabla x^{p}, x^{p} \nabla u_{i}\right\rangle=-\left\|u_{i}\right\|^{2}-\int\left\langle u_{i} \nabla x^{p}, x^{p} \nabla u_{i}\right\rangle,
$$

we obtain

$$
\int\left\langle u_{i} \nabla x^{p}, x^{p} \nabla u_{i}\right\rangle=-\frac{1}{2}\left\|u_{i}\right\|^{2} .
$$

According to (2.13) and (2.14), we have

$$
3\left\|\left\langle\nabla x^{p}, \nabla u_{i}\right\rangle\right\|^{2}-2 \Lambda_{i}\left\|\nabla q_{p i}\right\|^{2}=\frac{1}{2}-\frac{1}{2} \Lambda_{i}\left\|u_{i}\right\|^{2} .
$$

It finishes the proof of the lemma 2.2.

Lemma 2.3. For any $i$,

$$
\Lambda_{i} \sum_{p=1}^{n}\left\|\mathbf{w}_{p i}\right\|^{2} \geq(n-1)
$$

holds.

Proof. Since

$$
\nabla_{\beta}\left(x^{p} \nabla_{\alpha} u_{i}\right)-\nabla_{\alpha}\left(x^{p} \nabla_{\beta} u_{i}\right)=\nabla_{\beta} w_{p i}^{\alpha}-\nabla_{\alpha} w_{p i}^{\beta},
$$

where $w_{p i}^{\alpha}=x^{p} \nabla_{\alpha} u_{i}-\nabla_{\alpha} h_{p i}$ denotes the $\alpha$-th component of $\mathbf{w}_{p i}$, we infer, from $\operatorname{div}\left(\mathbf{w}_{p i}\right)=0$

$$
\begin{aligned}
\left\|\nabla \mathbf{w}_{p i}\right\|^{2} & =\sum_{\alpha, \beta=1}^{n}\left\|\nabla_{\alpha} w_{p i}^{\beta}\right\|^{2} \\
& =\frac{1}{2} \sum_{\alpha, \beta=1}^{n}\left\|\nabla_{\beta} w_{p i}^{\alpha}-\nabla_{\alpha} w_{p i}^{\beta}\right\|^{2}+\left\|\operatorname{div}\left(\mathbf{w}_{p i}\right)\right\|^{2} \\
& =\frac{1}{2} \sum_{\alpha, \beta=1}^{n}\left\|\nabla_{\beta}\left(x^{p} \nabla_{\alpha} u_{i}\right)-\nabla_{\alpha}\left(x^{p} \nabla_{\beta} u_{i}\right)\right\|^{2} \\
& =1-\left\|\nabla_{p} u_{i}\right\|^{2} .
\end{aligned}
$$

Furthermore, we have

$$
\begin{aligned}
& \Delta w_{p i}^{\alpha} \\
& =\Delta\left(x^{p} \nabla_{\alpha} u_{i}-\nabla_{\alpha} h_{p i}\right) \\
& =\Delta\left(x^{p} \nabla_{\alpha} u_{i}\right)-\nabla_{\alpha}\left(\operatorname{div}\left(\nabla h_{p i}\right)\right) \\
& =\Delta\left(x^{p} \nabla_{\alpha} u_{i}\right)-\nabla_{\alpha}\left(\operatorname{div}\left(x^{p} \nabla u_{i}\right)\right) \\
& =\nabla_{p} \nabla_{\alpha} u_{i}-\nabla_{\alpha} x^{p} \Delta u_{i} .
\end{aligned}
$$

Thus, we obtain

$$
\Delta \mathbf{w}_{p i}=\nabla\left\langle\nabla x^{p}, \nabla u_{i}\right\rangle-\Delta u_{i} \nabla x^{p}
$$


For any positive constant $\epsilon_{i}$, we have

$$
\begin{aligned}
& \left\|\nabla \mathbf{w}_{p i}\right\|^{2}=-\int\left\langle\mathbf{w}_{p i}, \Delta \mathbf{w}_{p i}\right\rangle \\
& =-\int\left\langle\mathbf{w}_{p i}, \nabla\left\langle\nabla x^{p}, \nabla u_{i}\right\rangle-\Delta u_{i} \nabla x^{p}\right\rangle \\
& \leq \frac{\epsilon_{i}}{2}\left\|\mathbf{w}_{p i}\right\|^{2}+\frac{1}{2 \epsilon_{i}}\left\|\nabla\left\langle\nabla x^{p}, \nabla u_{i}\right\rangle-\Delta u_{i} \nabla x^{p}\right\|^{2} .
\end{aligned}
$$

Since, from (2.17),

$$
\sum_{p=1}^{n}\left\|\nabla \mathbf{w}_{p i}\right\|^{2}=n-1, \quad \sum_{p=1}^{n}\left\|\nabla\left\langle\nabla x^{p}, \nabla u_{i}\right\rangle\right\|=\Lambda_{i},
$$

by taking sum on $p$ from 1 to $n$ for (2.19), we have

$$
(n-1) \leq \frac{\epsilon_{i}}{2} \sum_{p=1}^{n}\left\|\mathbf{w}_{p i}\right\|^{2}+\frac{n-1}{2 \epsilon_{i}} \Lambda_{i}
$$

Putting

$$
\epsilon_{i}=\sqrt{\frac{(n-1) \Lambda_{i}}{\sum_{p=1}^{n}\left\|\mathbf{w}_{p i}\right\|^{2}}}
$$

we obtain

$$
\Lambda_{i} \sum_{p=1}^{n}\left\|\mathbf{w}_{p i}\right\|^{2} \geq(n-1) .
$$

It completes the proof of the lemma 2.3.

Proof of Theorem 1.1. Since $\varphi_{p i}$ is a trial function, from the Rayleigh-Ritz inequality, we have

$$
\Lambda_{k+1}\left\|\nabla \varphi_{p i}\right\|^{2} \leq \int \varphi_{p i} \Delta^{2} \varphi_{p i}=-\int \nabla \varphi_{p i} \cdot \nabla(\Delta \varphi)_{p i} .
$$

By making use of the same arguments as in Cheng and Yang [7, we have, for any $p$ and $i$,

$$
\begin{gathered}
\left(\Lambda_{k+1}-\Lambda_{i}\right)\left\|\nabla \varphi_{p i}\right\|^{2} \leq 1+3\left\|\nabla_{p} u_{i}\right\|^{2}-\Lambda_{i}\left(\left\|u_{i}\right\|^{2}-\left\|\mathbf{w}_{p i}\right\|^{2}\right)+\sum_{j=1}^{k}\left(\Lambda_{i}-\Lambda_{j}\right) b_{p i j}^{2} \\
1+2 \sum_{j=1}^{k} b_{p i j} c_{p i j}=-2 \int_{\Omega}\left\langle\nabla \varphi_{p i}, \nabla\left\langle\nabla x^{p}, \nabla u_{i}\right\rangle\right\rangle
\end{gathered}
$$

where

$$
c_{p i j}=\int\left\langle\nabla\left\langle\nabla x^{p}, \nabla u_{i}\right\rangle, \nabla u_{j}\right\rangle=-c_{p j i} .
$$


Hence, we have, for any positive constant $\delta_{i}$,

$$
\begin{aligned}
& \left(\Lambda_{k+1}-\Lambda_{i}\right)^{2}\left(1+2 \sum_{j=1}^{k} b_{p i j} c_{p i j}\right) \\
& =\left(\Lambda_{k+1}-\Lambda_{i}\right)^{2} \int_{\Omega}-2\left\langle\nabla \varphi_{p i}, \nabla\left\langle\nabla x^{p}, \nabla u_{i}\right\rangle-\sum_{j=1}^{k} c_{p i j} \nabla u_{j}\right\rangle \\
& \leq \delta_{i}\left(\Lambda_{k+1}-\Lambda_{i}\right)^{3}\left\|\nabla \varphi_{p i}\right\|^{2}+\frac{1}{\delta_{i}}\left(\Lambda_{k+1}-\Lambda_{i}\right)\left(\left\|\nabla\left\langle\nabla x^{p}, \nabla u_{i}\right\rangle\right\|^{2}-\sum_{j=1}^{k} c_{p i j}^{2}\right) .
\end{aligned}
$$

From (2.21) and $\left\|u_{i}\right\|^{2}=\left\|\nabla q_{p i}\right\|^{2}+\left\|\mathbf{w}_{p i}\right\|^{2}$, we obtain

$$
\begin{aligned}
& \left(\Lambda_{k+1}-\Lambda_{i}\right)^{2}\left(1+2 \sum_{j=1}^{k} b_{p i j} c_{p i j}\right) \\
& \leq \delta_{i}\left(\Lambda_{k+1}-\Lambda_{i}\right)^{2}\left(1+3\left\|\nabla_{p} u_{i}\right\|^{2}-\Lambda_{i}\left\|\nabla q_{p i}\right\|^{2}+\sum_{j=1}^{k}\left(\Lambda_{i}-\Lambda_{j}\right) b_{p i j}^{2} \cdot\right) \\
& +\frac{1}{\delta_{i}}\left(\Lambda_{k+1}-\Lambda_{i}\right)\left(\left\|\nabla\left\langle\nabla x^{p}, \nabla u_{i}\right\rangle\right\|^{2}-\sum_{j=1}^{k} c_{p i j}^{2}\right) .
\end{aligned}
$$

By taking sum on $p$ from 1 to $n$, we derive

$$
\begin{aligned}
& \left(\Lambda_{k+1}-\Lambda_{i}\right)^{2}\left(n+2 \sum_{p=1}^{n} \sum_{j=1}^{k} b_{p i j} c_{p i j}\right) \\
& \leq \delta_{i}\left(\Lambda_{k+1}-\Lambda_{i}\right)^{2}\left(n+3-\Lambda_{i} \sum_{p=1}^{n}\left\|\nabla q_{p i}\right\|^{2}+\sum_{p=1}^{n} \sum_{j=1}^{k}\left(\Lambda_{i}-\Lambda_{j}\right) b_{p i j}^{2} \cdot\right) \\
& +\frac{1}{\delta_{i}}\left(\Lambda_{k+1}-\Lambda_{i}\right)\left(\Lambda_{i}-\sum_{p=1}^{n} \sum_{j=1}^{k} c_{p i j}^{2}\right)
\end{aligned}
$$

From the lemma 2.2, the lemma 2.3 and

$$
\left\|u_{i}\right\|^{2}=\left\|\nabla q_{p i}\right\|^{2}+\left\|\mathbf{w}_{p i}\right\|^{2}
$$

we infer

$$
\Lambda_{i} \sum_{p=1}^{n}\left\|\nabla q_{p i}\right\|^{2} \geq \frac{5}{3}
$$


Thus, we obtain, for any $i$,

$$
\begin{aligned}
& \left(\Lambda_{k+1}-\Lambda_{i}\right)^{2}\left(n+2 \sum_{p=1}^{n} \sum_{j=1}^{k} b_{p i j} c_{p i j}\right) \\
& \leq \delta_{i}\left(\Lambda_{k+1}-\Lambda_{i}\right)^{2}\left(n+\frac{4}{3}+\sum_{p=1}^{n} \sum_{j=1}^{k}\left(\Lambda_{i}-\Lambda_{j}\right) b_{p i j}^{2}\right) \\
& +\frac{1}{\delta_{i}}\left(\Lambda_{k+1}-\Lambda_{i}\right)\left(\Lambda_{i}-\sum_{p=1}^{n} \sum_{j=1}^{k} c_{p i j}^{2}\right) .
\end{aligned}
$$

By taking sum for $i$ from 1 to $k$ and noticing that $b_{p i j}$ is symmetric and $c_{p i j}$ is antisymmetric on $i, j$, we have

$$
\begin{aligned}
& n \sum_{i=1}^{k}\left(\Lambda_{k+1}-\Lambda_{i}\right)^{2}-2 \sum_{p=1}^{n} \sum_{i, j=1}^{k}\left(\Lambda_{k+1}-\Lambda_{i}\right)\left(\Lambda_{i}-\Lambda_{j}\right) b_{p i j} c_{p i j} \\
& \leq\left(n+\frac{4}{3}\right) \sum_{i=1}^{k} \delta_{i}\left(\Lambda_{k+1}-\Lambda_{i}\right)^{2}+\sum_{i=1}^{k} \frac{1}{\delta_{i}}\left(\Lambda_{k+1}-\Lambda_{i}\right) \Lambda_{i} \\
& -\sum_{p=1}^{n} \sum_{i, j=1}^{k} \delta_{i}\left(\Lambda_{k+1}-\Lambda_{i}\right)\left(\Lambda_{i}-\Lambda_{j}\right)^{2} b_{p i j}^{2}-\sum_{i, j=1}^{k} \frac{1}{\delta_{i}}\left(\Lambda_{k+1}-\Lambda_{i}\right) c_{p i j}^{2} \\
& +\sum_{p=1}^{n} \sum_{i, j=1}^{k} \delta_{i}\left(\Lambda_{k+1}-\Lambda_{i}\right)\left(\Lambda_{i}-\Lambda_{j}\right)^{2} b_{p i j}^{2} \\
& +\sum_{p=1}^{n} \sum_{i, j=1}^{k} \delta_{i}\left(\Lambda_{k+1}-\Lambda_{i}\right)^{2}\left(\Lambda_{i}-\Lambda_{j}\right) b_{p i j}^{2} .
\end{aligned}
$$

Since, for a non-increasing monotone sequence $\left\{\delta_{i}\right\}_{i=1}^{k}$,

$$
\begin{aligned}
& \sum_{p=1}^{n} \sum_{i, j=1}^{k} \delta_{i}\left(\Lambda_{k+1}-\Lambda_{i}\right)\left(\Lambda_{i}-\Lambda_{j}\right)^{2} b_{p i j}^{2}+\sum_{p=1}^{n} \sum_{i, j=1}^{k} \delta_{i}\left(\Lambda_{k+1}-\Lambda_{i}\right)^{2}\left(\Lambda_{i}-\Lambda_{j}\right) b_{p i j}^{2} \\
& =\frac{1}{2} \sum_{p=1}^{n} \sum_{i, j=1}^{k}\left(\Lambda_{k+1}-\Lambda_{i}\right)\left(\Lambda_{k+1}-\Lambda_{j}\right)\left(\Lambda_{i}-\Lambda_{j}\right)\left(\delta_{i}-\delta_{j}\right) b_{p i j}^{2} \leq 0 .
\end{aligned}
$$

We conclude from (2.26) and the above formula, for a non-increasing monotone sequence $\left\{\delta_{i}\right\}_{i=1}^{k}$,

$$
n \sum_{i=1}^{k}\left(\Lambda_{k+1}-\Lambda_{i}\right)^{2} \leq\left(n+\frac{4}{3}\right) \sum_{i=1}^{k} \delta_{i}\left(\Lambda_{k+1}-\Lambda_{i}\right)^{2}+\sum_{i=1}^{k} \frac{1}{\delta_{i}}\left(\Lambda_{k+1}-\Lambda_{i}\right) \Lambda_{i} .
$$

In particular, putting

$$
\delta_{i}=\sqrt{\frac{\sum_{i=1}^{k}\left(\Lambda_{k+1}-\Lambda_{i}\right) \Lambda_{i}}{\left(n+\frac{4}{3}\right) \sum_{i=1}^{k}\left(\Lambda_{k+1}-\Lambda_{i}\right)^{2}}}
$$


for any $i$, we obtain

$$
\sum_{i=1}^{k}\left(\Lambda_{k+1}-\Lambda_{i}\right)^{2} \leq \frac{4\left(n+\frac{4}{3}\right)}{n^{2}} \sum_{i=1}^{k}\left(\Lambda_{k+1}-\Lambda_{i}\right) \Lambda_{i} .
$$

This finishes the proof of the theorem 1.1.

Remark 2.1. If one can prove, for any $i$,

$$
\Lambda_{i} \sum_{p=1}^{n}\left\|\nabla q_{p i}\right\|^{2} \geq 3
$$

one will infer

$$
\sum_{i=1}^{k}\left(\Lambda_{k+1}-\Lambda_{i}\right)^{2} \leq \frac{4}{n} \sum_{i=1}^{k}\left(\Lambda_{k+1}-\Lambda_{i}\right) \Lambda_{i}
$$

which solves the conjecture.

\section{Proof of the theorem 1.2}

For the unit sphere

$$
S^{n}(1)=\left\{\left(x^{1}, x^{2}, \cdots, x^{n+1}\right) \in \mathbf{R}^{n+1} ; \sum_{i=1}^{n+1}\left(x^{p}\right)^{2}=1\right\},
$$

we denote the induced metric on $S^{n}(1)$ by the canonical metric $\langle\cdot, \cdot\rangle$ on $\mathbf{R}^{n+1}$ also. For any $p$, we have

$$
\nabla_{i} \nabla_{j} x^{p}=-g_{i j} x^{p}, \quad \Delta x^{p}=-n x^{p},
$$

where $g_{i j}$ denotes components of the metric tensor of $S^{n}(1)$. Let $u_{i}$ be the $i$-th orthonormal eigenfunction of the buckling problem (1.2) corresponding to the eigenvalue $\Lambda_{i}$, namely, $u_{i}$ satisfies

$$
\left\{\begin{array}{l}
\Delta^{2} u_{i}=-\Lambda_{i} \Delta u_{i} \quad \text { in } \Omega \\
\left.u_{i}\right|_{\partial \Omega}=\left.\frac{\partial u_{i}}{\partial \nu}\right|_{\partial \Omega}=0 \\
\left(u_{i}, u_{j}\right)_{D}=\int_{\Omega}\left\langle\nabla u_{i}, \nabla u_{j}\right\rangle=\delta_{i j}
\end{array}\right.
$$

For constructing trial functions, we use the same notations as in the section 2 . We would like to remark that vector-valued functions in this section have $n+1$ components. Although the orders of differentiations of functions in the Euclidean space can be exchanged freely, we must do it very carefully for the covariant differentiations of functions in the case of the unit sphere.

Since $x^{p}$ for $p=1,2, \cdots, n+1$ is a coordinate function of $\mathbf{R}^{n+1}$, for the vectorvalued function $x^{p} \nabla u_{i}, i=1, \ldots, k$, we decompose it into

$$
x^{p} \nabla u_{i}=\nabla h_{p i}+\mathbf{w}_{p i},
$$

where $h_{p i} \in H_{2, D}^{2}(\Omega)$ and $\nabla h_{p i}$ is the projection of $x^{p} \nabla u_{i}$ onto $\mathbf{H}_{1, D}^{2}(\Omega)$ and $\mathbf{w}_{p i} \perp$ $H_{1, D}^{2}(\Omega)$. Thus, we have, for any function $h \in C^{1}(\Omega) \cap L^{2}(\Omega)$,

$$
\left(\mathbf{w}_{p i}, \nabla h\right)=0 \text {. }
$$


Hence, $\mathbf{w}_{p i}$ satisfies

$$
\left\{\begin{array}{l}
\left.\mathbf{w}_{p i}\right|_{\partial \Omega}=0 \\
\left\|\operatorname{div} \mathbf{w}_{p i}\right\|^{2}=0 .
\end{array}\right.
$$

We define function $\varphi_{p i}$ by

$$
\varphi_{p i}=h_{p i}-\sum_{j=1}^{k} b_{p i j} u_{j}
$$

where

$$
b_{p i j}=\int x^{p}\left\langle\nabla u_{i}, \nabla u_{j}\right\rangle=b_{p j i} .
$$

It is easy to check that $\varphi_{p i}$ satisfies

$$
\left.\varphi_{p i}\right|_{\partial \Omega}=\left.\frac{\partial \varphi_{p i}}{\partial \nu}\right|_{\partial \Omega}=0 \text { and }\left(\varphi_{p i}, u_{j}\right)_{D}=\left(\nabla \varphi_{p i}, \nabla u_{j}\right)=0
$$

for any $j=1,2, \cdots, k$, that is, $\varphi_{p i}$ is a trial function. Since $\sum_{p=1}^{n+1}\left(x^{p}\right)^{2}=1$, from (3.3), we have, for any $i$,

$$
1=\sum_{p=1}^{n+1}\left\|\nabla h_{p i}\right\|^{2}+\sum_{p=1}^{n+1}\left\|\mathbf{w}_{p i}\right\|^{2} .
$$

Lemma 3.1. For any $i$, we have

$$
\sum_{p=1}^{n+1}\left\|\mathbf{w}_{p i}\right\|^{2} \leq \frac{\Lambda_{i}-(n-1)}{\Lambda_{i}-(n-2)} .
$$

Proof. From $\sum_{p=1}^{n+1}\left(x^{p}\right)^{2}=1$, we have

$$
\begin{aligned}
1 & =\sum_{p=1}^{n+1}\left\|\left\langle\nabla x^{p}, \nabla u_{i}\right\rangle\right\|^{2} \\
& =-\sum_{p=1}^{n+1} \int x^{p} \operatorname{div}\left\{\left\langle\nabla x^{p}, \nabla u_{i}\right\rangle \nabla u_{i}\right\} \\
& =-\sum_{p=1}^{n+1} \int x^{p}\left\langle\nabla x^{p}, \nabla u_{i}\right\rangle \Delta u_{i}-\sum_{p=1}^{n+1} \int\left\langle x^{p} \nabla u_{i}, \nabla\left\langle\nabla x^{p}, \nabla u_{i}\right\rangle\right\rangle \\
& =-\sum_{p=1}^{n+1} \int\left\langle\nabla h_{p i}, \nabla\left\langle\nabla x^{p}, \nabla u_{i}\right\rangle\right\rangle .
\end{aligned}
$$

For any positive constant $\epsilon_{i}$, we have

$$
1 \leq \epsilon_{i} \sum_{p=1}^{n+1}\left\|\nabla h_{p i}\right\|^{2}+\frac{1}{4 \epsilon_{i}} \sum_{p=1}^{n+1}\left\|\nabla\left\langle\nabla x^{p}, \nabla u_{i}\right\rangle\right\|^{2}
$$


According to the following Bochner formula for a smooth function $f$ :

$$
\begin{aligned}
\frac{1}{2} \Delta|\nabla f|^{2} & =\left|\nabla^{2} f\right|^{2}+\langle\nabla f, \nabla(\Delta f)\rangle+\operatorname{Ric}(\nabla f, \nabla f) \\
& =\left|\nabla^{2} f\right|^{2}+\langle\nabla f, \nabla(\Delta f)\rangle+(n-1)|\nabla f|^{2},
\end{aligned}
$$

where Ric and $\nabla^{2} f$ denote the Ricci tensor of $S^{n}(1)$ and the Hessian of $f$, respectively, we can derive, from (3.1) and by making use of a direct computation,

$$
\Delta\left\langle\nabla x^{p}, \nabla u_{i}\right\rangle=-2 x^{p} \Delta u_{i}+\left\langle\nabla x^{p}, \nabla\left(\Delta u_{i}\right)\right\rangle+(n-2)\left\langle\nabla x^{p}, \nabla u_{i}\right\rangle .
$$

Hence, we have

$$
\begin{aligned}
& \sum_{p=1}^{n+1}\left\|\nabla\left\langle\nabla x^{p}, \nabla u_{i}\right\rangle\right\|^{2} \\
& =-\sum_{p=1}^{n+1} \int\left\langle\nabla x^{p}, \nabla u_{i}\right\rangle \Delta\left\langle x^{p}, \nabla u_{i}\right\rangle \\
& =-\sum_{p=1}^{n+1} \int\left\langle\nabla x^{p}, \nabla u_{i}\right\rangle\left\{-2 x^{p} \Delta u_{i}+\left\langle\nabla x^{p}, \nabla\left(\Delta u_{i}\right)\right\rangle+(n-2)\left\langle\nabla x^{p}, \nabla u_{i}\right\rangle\right\} \\
& =-\sum_{p=1}^{n+1}\left\{\int\left\langle\nabla x^{p}, \nabla u_{i}\right\rangle\left\langle\nabla x^{p}, \nabla\left(\Delta u_{i}\right)\right\rangle+(n-2)\left\langle\nabla x^{p}, \nabla u_{i}\right\rangle^{2}\right\} \\
& =-\int\left\langle\nabla u_{i}, \nabla\left(\Delta u_{i}\right)\right\rangle-(n-2)\left\|\nabla u_{i}\right\| 2 \\
& =\Lambda_{i}-(n-2),
\end{aligned}
$$

that is,

$$
\sum_{p=1}^{n+1}\left\|\nabla\left\langle\nabla x^{p}, \nabla u_{i}\right\rangle\right\|^{2}=\Lambda_{i}-(n-2) .
$$

Here we have used

$$
\sum_{p=1}^{n+1} \int\left\langle\nabla x^{p}, \nabla u_{i}\right\rangle\left\langle\nabla x^{p}, \nabla\left(\Delta u_{i}\right)\right\rangle=\int\left\langle\nabla u_{i}, \nabla\left(\Delta u_{i}\right)\right\rangle .
$$

Therefore, from (3.9), we obtain

$$
1 \leq \epsilon_{i} \sum_{p=1}^{n+1}\left\|\nabla h_{p i}\right\|^{2}+\frac{1}{4 \epsilon_{i}}\left(\Lambda_{i}-(n-2)\right)
$$

From (3.7), we have

$$
1+\epsilon_{i} \sum_{p=1}^{n+1}\left\|\mathbf{w}_{p i}\right\|^{2} \leq \epsilon_{i}+\frac{1}{4 \epsilon_{i}}\left(\Lambda_{i}-(n-2)\right) .
$$

Taking

$$
\epsilon_{i}=\frac{\Lambda_{i}-(n-2)}{2}
$$

we complete the proof of the lemma 3.1. 
Proof of Theorem 1.2. By making use of the trial function $\varphi_{p i}$ and the same argumants as in Wang and Xia [16], we have, for any $p$ and $i$,

$$
\left(\Lambda_{k+1}-\Lambda_{i}\right)\left\|\nabla \varphi_{p i}\right\|^{2} \leq P_{p i}+\left\|\left\langle\nabla x^{p}, \nabla u_{i}\right\rangle\right\|^{2}+\Lambda_{i}\left\|\mathbf{w}_{p i}\right\|^{2}+\sum_{j=1}^{k}\left(\Lambda_{i}-\Lambda_{j}\right) b_{p i j}^{2}
$$

where

$$
P_{p i}=\int\left\langle\nabla\left(x^{p}\right)^{2}, u_{i} \nabla\left(\Delta u_{i}\right)+\Lambda_{i} u_{i} \nabla u_{i}\right\rangle
$$

Defining

$$
\begin{gathered}
Z_{p i}=\nabla\left\langle\nabla x^{p}, \nabla u_{i}\right\rangle-\frac{n-2}{2} x^{p} \nabla u_{i}, \\
c_{p i j}=\int\left\langle\nabla u_{j}, Z_{p i}\right\rangle=-c_{p j i}
\end{gathered}
$$

has been proved in Wang and Xia [16]. Since

$$
\begin{aligned}
\gamma_{p i} & =-2 \int\left\langle x^{p} \nabla u_{i}, Z_{p i}\right\rangle \\
& =-2 \int\left\langle\nabla h_{p i}+\mathbf{w}_{p i}, Z_{p i}\right\rangle \\
& =-2 \int\left\langle\nabla \varphi_{p i}+\sum_{j=1}^{k} b_{p i j} \nabla u_{j}+\mathbf{w}_{p i}, Z_{p i}\right\rangle \\
& =-2 \int\left\langle\nabla \varphi_{p i}, Z_{p i}-\sum_{j=1}^{k} c_{p i j} \nabla u_{j}\right\rangle-2 \sum_{j=1}^{k} b_{p i j} c_{p i j}+(n-2)\left\|\mathbf{w}_{p i}\right\|^{2},
\end{aligned}
$$

we have

$$
\gamma_{p i}+2 \sum_{j=1}^{k} b_{p i j} c_{p i j}=-2 \int\left\langle\nabla \varphi_{p i}, Z_{p i}-\sum_{j=1}^{k} c_{p i j} \nabla u_{j}\right\rangle+(n-2)\left\|\mathbf{w}_{p i}\right\|^{2} .
$$

Hence, for any positive constant $\delta_{i}$, we have, according to (3.12),

$$
\begin{aligned}
& \left(\Lambda_{k+1}-\Lambda_{i}\right)^{2}\left(\gamma_{p i}+2 \sum_{j=1}^{k} b_{p i j} c_{p i j}\right)-(n-2)\left(\Lambda_{k+1}-\Lambda_{i}\right)^{2}\left\|\mathbf{w}_{p i}\right\|^{2} \\
& \leq \delta_{i}\left(\Lambda_{k+1}-\Lambda_{i}\right)^{3}\left\|\nabla \varphi_{p i}\right\|^{2}+\frac{1}{\delta_{i}}\left(\Lambda_{k+1}-\Lambda_{i}\right)\left(\left\|Z_{p i}\right\|^{2}-\sum_{j=1}^{k} c_{p i j}^{2}\right) \\
& \leq \delta_{i}\left(\Lambda_{k+1}-\Lambda_{i}\right)^{2}\left\{P_{p i}+\left\|\left\langle\nabla x^{p}, \nabla u_{i}\right\rangle\right\|^{2}+\Lambda_{i}\left\|\mathbf{w}_{p i}\right\|^{2}+\sum_{j=1}^{k}\left(\Lambda_{i}-\Lambda_{j}\right) b_{p i j}^{2}\right\} \\
& +\frac{1}{\delta_{i}}\left(\Lambda_{k+1}-\Lambda_{i}\right)\left(\left\|Z_{p i}\right\|^{2}-\sum_{j=1}^{k} c_{p i j}^{2}\right) .
\end{aligned}
$$


By taking sum on $p$ from 1 to $n$, we derive

$$
\begin{aligned}
& \left(\Lambda_{k+1}-\Lambda_{i}\right)^{2} \sum_{p=1}^{n+1}\left(\gamma_{p i}+2 \sum_{j=1}^{k} b_{p i j} c_{p i j}\right)-(n-2)\left(\Lambda_{k+1}-\Lambda_{i}\right)^{2} \sum_{p=1}^{n+1}\left\|\mathbf{w}_{p i}\right\|^{2} \\
& \leq \delta_{i}\left(\Lambda_{k+1}-\Lambda_{i}\right)^{2} \sum_{p=1}^{n+1}\left\{P_{p i}+\left\|\left\langle\nabla x^{p}, \nabla u_{i}\right\rangle\right\|^{2}\right. \\
& \left.+\Lambda_{i}\left\|\mathbf{w}_{p i}\right\|^{2}+\sum_{j=1}^{k}\left(\Lambda_{i}-\Lambda_{j}\right) b_{p i j}^{2}\right\} \\
& +\frac{1}{\delta_{i}}\left(\Lambda_{k+1}-\Lambda_{i}\right) \sum_{p=1}^{n+1}\left(\left\|Z_{p i}\right\|^{2}-\sum_{j=1}^{k} c_{p i j}^{2}\right)
\end{aligned}
$$

Since

$$
\begin{aligned}
\gamma_{p i} & =-2 \int\left\langle x^{p} \nabla u_{i}, Z_{p i}\right\rangle \\
& =-2 \int\left\langle x^{p} \nabla u_{i}, \nabla\left\langle\nabla x^{p}, \nabla u_{i}\right\rangle-\frac{n-2}{2} x^{p} \nabla u_{i}\right\rangle \\
& =2 \int\left\langle\nabla x^{p}, \nabla u_{i}\right\rangle^{2}+2 \int \Delta u_{i}\left\langle x^{p} \nabla x^{p}, \nabla u_{i}\right\rangle+(n-2) \int\left(x^{p}\right)^{2}\left\langle\nabla u_{i}, \nabla u_{i}\right\rangle,
\end{aligned}
$$

we have

$$
\sum_{p=1}^{n+1} \gamma_{p i}=n
$$

From the definition of $Z_{p i}$, we have

$$
\begin{aligned}
& \sum_{p=1}^{n+1}\left\|Z_{p i}\right\|^{2} \\
& =\sum_{p=1}^{n+1} \int\left|\nabla\left\langle\nabla x^{p}, \nabla u_{i}\right\rangle-\frac{n-2}{2} x^{p} \nabla u_{i}\right|^{2} \\
& =\sum_{p=1}^{n+1}\left\{\left\|\nabla\left\langle\nabla x^{p}, \nabla u_{i}\right\rangle\right\|^{2}-(n-2) \int\left\langle\nabla\left\langle\nabla x^{p}, \nabla u_{i}\right\rangle, x^{p} \nabla u_{i}\right\rangle+\frac{(n-2)^{2}}{4}\left\|x^{p} \nabla u_{i}\right\|^{2}\right\} \\
& =\Lambda_{i}+\frac{(n-2)^{2}}{4}(\text { from }(3.11)) .
\end{aligned}
$$

Since $P_{p i}=\int\left\langle\nabla\left(x^{p}\right)^{2}, u_{i} \nabla\left(\Delta u_{i}\right)+\Lambda_{i} u_{i} \nabla u_{i}\right\rangle$, we have

$$
\sum_{p=1}^{n+1} P_{p i}=0 .
$$


From the lemma 3.1 and (3.14), we obtain

$$
\begin{aligned}
& \left(\Lambda_{k+1}-\Lambda_{i}\right)^{2}\left(n+2 \sum_{p=1}^{n+1} \sum_{j=1}^{k} b_{p i j} c_{p i j}\right)-(n-2)\left(\Lambda_{k+1}-\Lambda_{i}\right)^{2} \frac{\Lambda_{i}-(n-1)}{\Lambda_{i}-(n-2)} \\
& \leq \delta_{i}\left(\Lambda_{k+1}-\Lambda_{i}\right)^{2}\left\{1+\Lambda_{i} \frac{\Lambda_{i}-(n-1)}{\Lambda_{i}-(n-2)}+\sum_{p=1}^{n+1} \sum_{j=1}^{k}\left(\Lambda_{i}-\Lambda_{j}\right) b_{p i j}^{2}\right\} \\
& +\frac{1}{\delta_{i}}\left(\Lambda_{k+1}-\Lambda_{i}\right)\left(\Lambda_{i}+\frac{(n-2)^{2}}{4}\right)-\frac{1}{\delta_{i}}\left(\Lambda_{k+1}-\Lambda_{i}\right) \sum_{p=1}^{n+1} \sum_{j=1}^{k} c_{p i j}^{2}
\end{aligned}
$$

that is,

$$
\begin{aligned}
& 2\left(\Lambda_{k+1}-\Lambda_{i}\right)^{2}+(n-2) \frac{\left(\Lambda_{k+1}-\Lambda_{i}\right)^{2}}{\Lambda_{i}-(n-2)} \\
& \leq \delta_{i}\left(\Lambda_{k+1}-\Lambda_{i}\right)^{2}\left\{\Lambda_{i}-\frac{(n-2)}{\Lambda_{i}-(n-2)}\right\}+\frac{1}{\delta_{i}}\left(\Lambda_{k+1}-\Lambda_{i}\right)\left(\Lambda_{i}+\frac{(n-2)^{2}}{4}\right) \\
& -2\left(\Lambda_{k+1}-\Lambda_{i}\right)^{2} \sum_{p=1}^{n+1} \sum_{j=1}^{k} b_{p i j} c_{p i j}+\delta_{i}\left(\Lambda_{k+1}-\Lambda_{i}\right)^{2} \sum_{p=1}^{n+1} \sum_{j=1}^{k}\left(\Lambda_{i}-\Lambda_{j}\right) b_{p i j}^{2} \\
& -\frac{1}{\delta_{i}}\left(\Lambda_{k+1}-\Lambda_{i}\right) \sum_{p=1}^{n+1} \sum_{j=1}^{k} c_{p i j}^{2} .
\end{aligned}
$$

Since, for a non-increasing monotone sequence $\left\{\delta_{i}\right\}_{i=1}^{k}$,

$$
\begin{aligned}
& \sum_{p=1}^{n} \sum_{i, j=1}^{k} \delta_{i}\left(\Lambda_{k+1}-\Lambda_{i}\right)\left(\Lambda_{i}-\Lambda_{j}\right)^{2} b_{p i j}^{2}+\sum_{p=1}^{n} \sum_{i, j=1}^{k} \delta_{i}\left(\Lambda_{k+1}-\Lambda_{i}\right)^{2}\left(\Lambda_{i}-\Lambda_{j}\right) b_{p i j}^{2} \\
& =\frac{1}{2} \sum_{p=1}^{n} \sum_{i, j=1}^{k}\left(\Lambda_{k+1}-\Lambda_{i}\right)\left(\Lambda_{k+1}-\Lambda_{j}\right)\left(\Lambda_{i}-\Lambda_{j}\right)\left(\delta_{i}-\delta_{j}\right) b_{p i j}^{2} \leq 0
\end{aligned}
$$

and

$$
\begin{aligned}
& -2 \sum_{i=1}^{k}\left(\Lambda_{k+1}-\Lambda_{i}\right)^{2} \sum_{p=1}^{n+1} \sum_{j=1}^{k} b_{p i j} c_{p i j}-\sum_{i=1}^{k} \delta_{i}\left(\Lambda_{k+1}-\Lambda_{i}\right) \sum_{p=1}^{n} \sum_{j=1}^{k}\left(\Lambda_{i}-\Lambda_{j}\right)^{2} b_{p i j}^{2} \\
& -\sum_{i=1}^{k} \frac{1}{\delta_{i}}\left(\Lambda_{k+1}-\Lambda_{i}\right) \sum_{p=1}^{n+1} \sum_{j=1}^{k} c_{p i j}^{2} \\
& =-\sum_{p=1}^{n} \sum_{i, j=1}^{k}\left(\sqrt{\delta_{i}\left(\Lambda_{k+1}-\Lambda_{i}\right)}\left(\Lambda_{i}-\Lambda_{j}\right) b_{p i j}-\frac{1}{\sqrt{\delta_{i}}} \sqrt{\left(\Lambda_{k+1}-\Lambda_{i}\right)} c_{p i j}\right)^{2} \leq 0,
\end{aligned}
$$


by taking sum on $i$ from 1 to $k$ for (3.15), we obtain

$$
\begin{aligned}
& 2 \sum_{i=1}^{k}\left(\Lambda_{k+1}-\Lambda_{i}\right)^{2}+(n-2) \sum_{i=1}^{k} \frac{\left(\Lambda_{k+1}-\Lambda_{i}\right)^{2}}{\Lambda_{i}-(n-2)} \\
& \leq \sum_{i=1}^{k} \delta_{i}\left(\Lambda_{k+1}-\Lambda_{i}\right)^{2}\left\{\Lambda_{i}-\frac{(n-2)}{\Lambda_{i}-(n-2)}\right\} \\
& +\sum_{i=1}^{k} \frac{1}{\delta_{i}}\left(\Lambda_{k+1}-\Lambda_{i}\right)\left(\Lambda_{i}+\frac{(n-2)^{2}}{4}\right) .
\end{aligned}
$$

It completes the proof of the theorem 1.2.

\section{REFERENCES}

[1] M. S. Ashbaugh, Isoperimetric and universal inequalities for eigenvalues, in Spectral theory and geometry (Edinburgh, 1998), E. B. Davies and Yu Safarov eds., London Math. Soc. Lecture Notes, vol. 273, Cambridge Univ. Press, Cambridge, 1999, pp. 95-139.

[2] M. S. Ashbaugh, On universal inequalities for the low eigenvalues of the buckling problem. Partial differential equations and inverse problems, 13-31, Contemp. Math., 362, Amer. Math. Soc., Providence, RI, 2004.

[3] M. S. Ashbaugh and L. Hermi, A unified approach to universal inequalities for eigenvalues of elliptic operators, Pacific J. Math. 217 (2004), 201-219.

[4] D. Chen and Q. -M. Cheng, Extrinsic estimates for eigenvalues of the Laplace operator, J. Math. Soc. Japan 60 (2008), 325-339.

[5] Q. -M. Cheng, T. Ichikawa and S. Mametsuka, Estimates for eigenvalues of a clamped plate problem on Riemannian manifolds, to appear in J. Math. Soc. Japan.

[6] Q. -M. Cheng and H. C. Yang, Estimates on Eigenvalues of Laplacian, Math. Ann. 331 (2005), 445-460.

[7] Q. -M. Cheng and H. C. Yang, Universal bounds for eigenvalues of a buckling problem, Commn. Math. Phys., 262 (2006), 663-675.

[8] Q. -M. Cheng and H. C. Yang, Bounds on eigenvalues of Dirichlet Laplacian, Math. Ann., 337 (2007), 159-175.

[9] Q. -M. Cheng and H. C. Yang, Estimates for eigenvalues on Riemannian manifolds, to appear in J. Diff. Eqns., 2009.

[10] Q. -M. Cheng and H. C. Yang, Universal inequalities for eigenvalues of a clamped plate problem on a hyperbolic space, preprint 2007.

[11] A. El Soufi, E. M. Harrell II and S. Ilias, Universal inequalities for the eigenvalues of Laplace and Schrödinger operators on submanifolds, Trans. Amer. Math. Soc., 361 (2009), 2337-2350.

[12] E. M. Harrell II, Commutators, eigenvalue gaps and mean curvature in the theory of Schrödinger operators, Comm. Part. Diff. Eqns., 32 (2007), 401-413.

[13] G. N. Hile and R. Z. Yeh, Inequalities for eigenvalues of the biharmonic operator, Pacific J. Math. 112 (1984), 115-133.

[14] L. E. Payne, G. Polya and H. F. Weinberger, Sur le quotient de deux fréquences propres consécutives, Comptes Rendus Acad. Sci. Paris, 241 (1955), 917-919.

[15] L. E. Payne, G. Pólya and H. F. Weinberger, On the ratio of consecutive eigenvalues, J. Math. and Phys. 35 (1956), 289-298.

[16] Q. Wang and C. Xia, Universal inequalities for eigenvalues of the buckling problem on spherical domains, Commn. Math. and Phys. 270 (2007), 759-775.

[17] Q. Wang and C. Xia, Universal bounds for eigenvalues of the biharmonic operator on Riemannian manifolds, J. Funct. Anal., 245 (2007), 334-352. 
Qing-Ming Cheng: Department of Mathematics, Faculty of Science And EngiNeEring, SAgA University, SAgA 840-8502, JAPAn. E-MAIl: CHEng@MS.SAGA-U.AC.JP

Hongcang Yang: Academy of Mathematics and Systematical Sciences, CAS, BeiJING 100080, ChinA. E-MAIL: YANGHC2@NETEASE.COM 